•综述・

\title{
海鸟种群的人工招引与恢复技术及其应用
}

\author{
周 晓 ${ }^{1}$ 陈东东 ${ }^{1}$ Stephen W. Kress ${ }^{2}$ 陈水华 ${ }^{1,3^{*}}$ \\ 1 (浙江师范大学化学与生命科学学院, 浙江金华 321004) \\ 2 (Seabird Restoration Program, National Audubon Society, Ithaca, NY 14850, USA) \\ 3 (浙江自然博物馆, 杭州 310014)
}

\begin{abstract}
摘要: 全球多数海鸟种群处于受胁状态。针对这一状况, 鸟类学家根据海鸟的繁殖习性, 借助于人工招引技术恢 复海鸟的种群及营建适宜的栖息地。海鸟人工招引技术主要包括社群吸引技术和雉鸟转移技术两大类。社群吸引 技术是在人为选择和改造的栖息地内利用假鸟模型和声音回放吸引附近海鸟前来栖息的方法; 倠鸟转移是将恢复 种群的倠鸟转移到改造好的栖息地内, 利用海鸟的成长地依赖性, 促使其在繁殖期主动回到生长地繁殖。这两种 方法已在全世界16个国家和地区的64种海鸟，一共171多个招引项目中得到了应用。在具体应用中，应该因地制宜， 针对不同的海鸟种类和繁殖习性选择合适的方法, 可单独或结合使用。不论采用何种方法, 都应开展充分的前期 研究, 选择并营造适宜的繁殖栖息地, 严格控制天敌, 密切监测, 及时排除威胁因素, 确保繁殖成功。我国沿海繁 殖海鸟正面临多种威胁, 海鸟种群缩小和栖息地丧失严重, 海鸟人工招引技术的应用为我国沿海繁殖海鸟种群及 其栖息地的恢复带来了希望。
\end{abstract}

关键词：海鸟；繁殖栖息地；种群恢复；社群吸引技术；雏鸟转移

\section{A review of the use of active seabird restoration techniques}

\author{
Xiao Zhou ${ }^{1}$, Dongdong Chen ${ }^{1}$, Stephen W. Kress ${ }^{2}$, Shuihua Chen ${ }^{1,3^{*}}$ \\ 1 College of Chemistry and Life Sciences, Zhejiang Normal University, Jinhua, Zhejiang 321004, China \\ 2 Seabird Restoration Program, National Audubon Society, Ithaca, NY 14850, USA \\ 3 Zhejiang Museum of Natural History, Hangzhou 310014, China
}

\begin{abstract}
Most seabird species are threatened or endangered. To restore their populations and breeding habitats, ornithologists have developed two major active seabird restoration techniques: social attraction and chick translocation. With social attraction, decoys and acoustic vocalization playback are used to lure breeding seabirds to the restoration site. In chick translocation, the chicks of focal species are translocated and reared at the restoration site with the goal that they will return and breed at the release site. We conducted a worldwide search and found 171 projects conducted in 16 countries/regions to restore 64 seabird species. These techniques are used either independently or in combination, depending on the breeding biology of the target species. For both methods, managers are encouraged to understand the species' breeding ecology and preferred nesting habitat. Habitat management, predator control and close monitoring are key steps to assure the success of the restoration. The breeding seabirds of China's coast face many threats and their populations and habitats have decreased rapidly in recent decades. Active seabird restoration techniques can help to expand ranges and provide resilience to threats by starting new nesting colonies along the coast of China.
\end{abstract}

Key words: seabird; breeding habitat; population restoration; social attraction; chick translocation

\section{1 引言}

海鸟又称海洋鸟类，是对那些适应海洋环境,
能在咸水中受食的鸟类的总称(Schreiber \& Burger, 2001)。海鸟种类较多, 形态和行为多样。海鸟一般

收稿日期: 2016-12-30; 接受日期: 2017-03-20

基金项目: 国家自然科学基金(31572291)

* 通讯作者 Author for correspondence. E-mail: chensh@zmnh.com 
寿命较长, 繁殖较晚, 窝卵数较少; 多数具有集群 营巢的习性, 集群中的个体数量少则十多只, 多达 数百万只; 许多海鸟具有长途迁徙的习性; 部分远 洋种类, 除了繁殖季节外, 大部分时间都在外海活 动(Gaston, 2004)。海鸟类群的界定并不是很清晰。 狭义上, 海鸟只包括企鹅目和獲形目全部种类, 鹈 形目除蛇鹈外的全部种类, 以及行形目中的贼鸥 科、鸥科、燕鸥科、剪嘴鸥科和海雀科鸟类。广义 上, 海鸟有时也包括在海洋环境栖息的鶓鷉科、潜 鸟科、瓣蹼䂆科、鹭科、噮科和鸭科的部分种类 (Gaston, 2004)。大部分鸟类学家接受狭义的海鸟定 义。按照传统的鸟类分类系统, 全世界列入海鸟类 群的共有 4目14科78属335种(Schreiber \& Burger, 2001)。

由于人类活动所导致的食物减少、环境污染、 渔网误捕、捡食鸟蛋和捕捉幼鸟、外来物种引入和 栖息地受损等原因, 全球接近 $1 / 3$ 的海鸟种群濒临 灭绝(IUCN, 2009)。其中分布范围狭窄、因全球气 候变化和海水酸化造成栖息地面积缩减、质量下降 的海鸟种群的受胁状况尤其严重(Croxall et al, 2002; Frederiksen et al, 2004)。鸟类学家提出了海鸟种群 恢复计划来保护受胁海鸟。海鸟种群恢复计划是通 过人为的干预措施提升海鸟栖息环境的质量(Kress, 1998)。海鸟种群恢复的重要性不仅在于保护海鸟物 种和数量, 更重要的是恢复海洋生态系统的功能, 促进生态进程, 使海鸟栖息环境、海洋营养物质循 环和陆生生态系统相统一, 为共生物种创造更加适 宜的生存环境(Jones \& Kress, 2012)。成功的海鸟种 群恢复项目可以扩大受胁种群的生存范围, 恢复其 种群数量, 尤其是那些遭受人类活动和天敌捕食的 影响而濒临消失的种群(Jones et al, 2011)。

目前, 海鸟种群恢复基本上针对海鸟繁殖栖息 地和繁殖种群的恢复, 主要存在两大途径: 一是自 然恢复途径(passive seabird restoration), 即着眼于 营造和恢复海鸟曾经的适宜栖息地, 消除海鸟繁殖 岛屿上的威胁因素, 包括人为引入的哺乳动物, 特 别是鼠类等对海鸟繁殖具有严重威胁的外来有害 生物, 停止旅游、生产等人为活动, 修整栖息地以 适合海鸟繁殖等, 等候海鸟通过自然扩散和栖息地 选择重新回到原有的繁殖地。另一途径是人工招引 恢复(active seabird restoration), 即在恢复栖息地适 宜性的基础上, 辅以人工方法吸引海鸟前来栖息。
自然恢复的方法时间跨度很长, 往往从项目开始到 见效需要十几年甚至几十年时间。这样的项目周期 对于实施方来说是很大的挑战, 一方面需要做长期 的监测, 另一方面, 在这十多年和数十年的等候过 程中, 经过消除天敌和修整的栖息地可能还会发生 变化, 仍然需要进行长期的维护和监测(Nogales et al, 2004; Donlan \& Wilcox, 2007; Howald et al, 2007)。更大的困难还在于, 这样需要长期投入但难 以见效的项目很难争取到经费的支持。因而在世界 范围, 虽然有科学家尝试海鸟种群的自然恢复, 但 真正实施的很少(Jones et al, 2011)。而人工招引的方 法由于项目周期相对较短, 且效果明显, 而且在种 群恢复的同时, 也可进一步促进栖息地的维护和恢 复。因此, 这一方法在世界各地得到了普遍的应用。 鉴于上述原因, 本文主要针对海鸟种群的人工招引 和恢复技术及其应用进行介绍。

海鸟种群人工招引和恢复的尝试始于 20 世纪 70年代在美国缅因州海湾北极海鹦(Fratercula arctica)种群的再引入。该项目的最初目的是恢复那些 由于人类开发或外来种入侵而消失的种群(Kress, 1998), 进而应用到解决因鱼鸟冲突、栖息地受损而 需要迁移的燕鸥繁殖种群(Hasegawa \& Watkinson, 1982; Roby et al, 2002; Parker et al, 2007)。该方法目 前已经在世界范围得到了广泛的应用, 是海洋鸟类 种群人工恢复的重要途径。

近年来, 由于浙江非山列岛针对极危鸟类中华 凤头燕鸥(Thalasseus bernsteini)人工招引项目的成 功(陈水华和范忠勇, 2013), 以及三亚海鸥招引项目 的实施, 海鸟人工招引技术及其应用现状引起了广 泛关注 (http://www.sanyarb.com.cn/content/2016-07/ 24/content_224832.htm)。Jones和Kress (2012)对此前 世界范围的海鸟人工招引和恢复项目进行了较为 全面的介绍, 在此基础上, 我们通过文献查阅、专 家咨询等多种方式补充了最新资料, 全面介绍海鸟 种群人工招引和恢复技术及其应用, 为我国海鸟多 样性的研究、保护和管理提供借鉴。

\section{2 海鸟种群人工招引和恢复技术}

海鸟种群人工招引和恢复技术可分为雉鸟转 移技术(chick translocation)和社群吸引技术(social attraction) (表1)。雏鸟转移主要针对那些有强烈恋 家(出生地)倾向的海鸟, 这些海鸟一般不需要双亲 
表1 海鸟种群人工招引和恢复两种主要技术对照表

Table 1 Comparisons between the two major techniques of active seabird restoration

\begin{tabular}{|c|c|c|}
\hline & 雏鸟转移技术 Chick translocation & 社群吸引技术 Social attraction \\
\hline $\begin{array}{l}\text { 原理 } \\
\text { Overview }\end{array}$ & $\begin{array}{l}\text { 转移维鸟至目标岛屿, 人工饲育直到飞行离开。利用海鸟 } \\
\text { 对生长地的依恋倾向, 吸引这些倠鸟长大后回归目标岛屿 } \\
\text { 繁殖 } \\
\text { Transfer seabird nestlings to the target island, and rear them } \\
\text { until they fledge. The released birds may return to the restora- } \\
\text { tion site. This method is best for species that demonstrate } \\
\text { strong natal site philopatry. }\end{array}$ & $\begin{array}{l}\text { 在目标岛屿上布置模型诱饵和声音回放设备, 利用 } \\
\text { 集群海岛的社群吸引行为吸引附近海鸟前来栖息繁 } \\
\text { 殖 } \\
\text { Decoys and audio playback systems are deployed at the } \\
\text { restoration site to attract adult colonial seabirds, which } \\
\text { select their breeding site by using social information. }\end{array}$ \\
\hline $\begin{array}{l}\text { 技术要点 } \\
\text { Key techniques }\end{array}$ & $\begin{array}{l}\text { 维鸟人工抚育及野外放飞 } \\
\text { Chick rearing and release }\end{array}$ & $\begin{array}{l}\text { 假鸟的布设及声音回放设备的安放 } \\
\text { Decoys and audio playback system deployment }\end{array}$ \\
\hline $\begin{array}{l}\text { 适用范围 } \\
\text { Preferred species }\end{array}$ & $\begin{array}{l}\text { 具有恋家倾向的、易于人工饲养、穴居型海鸟, 如信天翁 } \\
\text { 和海燕等 } \\
\text { Species with strong natal site philopatry such as albatrosses } \\
\text { and storm-petrels }\end{array}$ & $\begin{array}{l}\text { 集群性较强, 倠鸟需双亲后期抚育的海鸟, 如燕鸥和 } \\
\text { 海鸥等 } \\
\text { Colonial breeding species with weak natal site philo- } \\
\text { patry such as terns and gulls }\end{array}$ \\
\hline $\begin{array}{l}\text { 局限性 } \\
\text { Limitations }\end{array}$ & $\begin{array}{l}\text { 需投入较大人力物力用于雉鸟饲育; 不恰当的饲育和运输 } \\
\text { 可能对维鸟造成伤害; 取得成功周期相对较长 } \\
\text { Labor intensive and expensive because of the need for chick } \\
\text { tending stewards; great care during transport and chick rear- } \\
\text { ing to reduce mortality to chicks; may take many years to } \\
\text { achieve restoration because most species do not breed until } \\
\text { they are five or more years old. }\end{array}$ & $\begin{array}{l}\text { 见效快, 繁殖群的形成有滚雪球效应, 但除了燕鸥和 } \\
\text { 海鸥等集群性较强的海鸟, 其他海鸟招引成功率相 } \\
\text { 对较低 } \\
\text { Colonization around decoys and audio speakers may } \\
\text { happen quickly as the first breeders help to advertise the } \\
\text { location of the colony, but a relatively lower success } \\
\text { rate for other species except for terns and gulls. }\end{array}$ \\
\hline 见效期 & 5-10年 & 1-5年 \\
\hline
\end{tabular}

抚育，因此易于人工饲养。踓鸟转移需要将幼雉迁 移到目标岛屿上, 人工饲育直至飞行离开。这些海 鸟会对成长的环境留下记忆, 有可能在性成熟后回 归成长地繁殖, 甚至带动其他同种鸟类的个体一起 过来, 从而达到人工招引的目的(Miskelly \& Gummer, 2013)。维鸟转移由于涉及倠鸟人工抚育和野外 放飞, 因而需要投入较大的人力和物力。而且一般 限于以鱼类或反刍食物为食的鸟类, 如信天翁和海 燕等。对于需要双亲后期抚育的鸟类, 比如燕鸥, 雏鸟转移的方法则不合适。

社群吸引技术是目前开展海鸟种群恢复的最 主要手段。它主要是通过吸引繁殖期的成鸟到人为 规划的栖息地进行筑巢繁殖(Jones \& Kress, 2012)。 由于全球 $95 \%$ 以上的海鸟都具有集群性, 这意味着 它们会被同种或其他生态习性相近的海鸟吸引而 聚集繁殖(Rolland et al, 1998), 先期到达栖息地的海 鸟就可以作为活的诱饵吸引更多的海鸟前来。社群 吸引技术通常将模型诱饵和声音回放设备相结合, 模型多用于昼行性的海岛, 声音则对昼行性和夜行 性海鸟都有吸引作用(Jones \& Kress, 2012)。

社群吸引技术应用比较广泛, 主要是利用海鸟 集群性的特点(Rolland et al, 1998), 在其繁殖期到 来之前, 选择一个合适的岛屿, 人为改造成适宜海 鸟繁殖的生境, 利用假鸟、假蛋、人造洞穴以及海
鸟繁殖期声音的回放吸引海鸟来此繁殖。这项技术 是由美国奥杜邦协会(National Audubon Society)的 Stephen Kress博士提出, 并首先应用到缅因湾招引 普通燕鸥(Sterna hirundo)和北极燕鸥(S. paradisaea) 的项目上(Jones \& Kress, 2012)。

社群吸引技术的具体步骤和方法如下:

(1)选择合适的岛屿。岛屿的选择和拟吸引的鸟 类种类有关。建议在该物种的潜在繁殖区或历史繁 殖区选择招引栖息地。在选择的岛屿上根据需要改 造栖息地的坡度、植被密度和高度等环境特征, 以 保证可以随时监控整个繁殖种群。在开始招引活动 之前, 应清除岛上所有对繁殖海鸟具有威胁的物种, 包括蛇、鼠等天敌。在招引区附近搭建隐蔽的监测 站, 在不惊扰海鸟的情况下进行持续的观察。

(2)招引栖息地的改造。监测人员根据海鸟繁殖 所需的生境对环境基质进行改造, 如土壤、岩石和 植被比例, 洞穴, 植被高度等。在已经改造好的栖 息地上布设假鸟模型、声音回放设备等, 引诱附近 岛屿栖息或经过的海岛。在监测营地观察整个繁殖 区是否存在监测盲区, 如果存在, 在监测盲区附近 安装视频监控设备, 以便于全面了解整个栖息地的 状况。

(3)招引栖息地的监控和保护。海鸟被引诱来此 繁殖后, 要保持持续监控, 制止非法捡蛋和登岛等 
人为干扰行为, 同时查看整个栖息地内及周围环境 是否有威胁海鸟繁殖的潜在因素。对海鸟整个繁殖 期的行为进行监控, 包括筑巢地点选择、求偶交配、 产卵孵化、抚养雏鸟以及种间竞争合作等行为。观 察岛上栖息地周围是否有其他海鸟存在并监测其 行为对招引海鸟繁殖是否有影响。根据岛上实际情 况随时清除对招引海鸟产生威胁的因素, 包括捕食 性动物的抓捕、猛禽的驱赶等。

(4)招引栖息地附近区域的调查。确认招引栖息 地附近是否还存在其他繁殖群体, 以进一步评估和 分析招引的效果。

\section{3 海鸟种群人工招引技术的应用}

截至2016年底，应用海鸟人工招引技术的国家 和地区有 16 个, 涉及64种海鸟、171个招引项目(表 2)。所招引的海鸟涵盖了 $19.1 \%$ 的海鸟种类, 其中 $41 \%$ 的种类濒临灭绝。在这些项目中, 18 个项目采用 了雏鸟转移法, 12 个项目只使用了假鸟, 19个只使 用了声音回放, 9个项目结合使用了雏鸟转移和声 音回放, 3 个项目结合了雏鸟转移和假鸟, 76 个项目 结合了假鸟和声音回放, 9个项目结合了声音回放 和人工洞穴。

在已知招引结果的 112 个项目中, 75 个项目取 得了成功。其中, 仅使用声音回放、雏鸟转移和假 鸟的项目，成功率分别为 $42 \%(n=19) 、 87.5 \%(n=8)$ 和 $44 \%(n=9)$ 。而使用多种方法结合的, 招引成功 率则可达 $80 \%$ 以上 $(\mathrm{n}=56)$ 。从鸟类类群来看, 不同 类群的人工招引的成功率也存在差异。獲科鸟类招 引成功的比例最高, 达 $81 \%(\mathrm{n}=16)$, 燕鸥科招引成 功率为 $71 \%(\mathrm{n}=55)$, 鸥科为 $64 \%(\mathrm{n}=11)$, 海燕科 $60 \%(\mathrm{n}=10)$, 扸鹚科 $37.5 \%(\mathrm{n}=8)$, 信天翁科 $33 \%$ $(n=3)$ 。

\section{4 典型案例}

\section{1 美国缅因州北极海财和燕鸥的招引工作}

历史上，位于美国东北部缅因州的Eastern Egg Rock岛是北极海鹦曾经栖息繁殖的岛屿, 但19世纪 末以后它们从这个岛屿上消失了(Kress, 1997)。 1973年Stephen Kress博士等人选择了这个岛屿作为 招引海鹦的地点, 期望它们可以重返这个岛栖息繁 殖。他们首先把幼年海鹦转移到Eastern Egg Rock 岛上饲育, 希望它们性成熟以后可以返回该岛进行 繁殖。12年间, 他们共转移了 954 只维鸟, 其中940 只进行饲育后成功离巢。4年后, 当年饲育的北极海 鹦开始回归。8年后, 也即1981年, 5 对北极海鹦来此 筑巢繁殖。这是近百年来首次飞来此地繁殖的海鹦 (Kress \& Nettleship, 1988)。他们随后又在岛上布置 了许多假鸟来吸引海鹦。截止到2009年已经有 123 对北极海鹦在Eastern Egg Rock岛上筑巢繁殖(Kress et al, 2009)。

北极海鹦并不是缅因湾唯一消失的鸟类。20世 纪初以前, 燕鸥在缅因湾的栖息地和数量是很多的, 但从1914年以来, 经过人类猎杀、天敌捕食和其他 鸥类竞争等因素的影响, 燕鸥种群濒临灭绝(Kress, 1997)。随着海鸟种群恢复项目的提出, 1980年当地 政府为恢复燕鸥种群, 决定在Eastern Egg Rock岛 开展燕鸥人工招引项目。与北极海鹦招引不同的是, 燕鸥的倠鸟不适合人工迁移和饲育, 必须选择新的 招引方法。这次他们尝试实施的是社群吸引技术, 这项技术也是由Stephen Kress博士发明的。他们首 先清除了岛上所有对燕鸥有威胁的捕食动物(Kress, 1983), 然后在岛上布设了假鸟模型和非攻击

表2 海鸟人工恢复项目在世界各地的应用和分布

Table 2 Distribution of active seabird restoration projects worldwide

\begin{tabular}{ll||ll}
\hline 国家/地区 Country/region & 项目数 Number of projects & 国家/地区 Country/region & 项目数 Number of projects \\
\hline 加拿大 Canada & 6 & 威尔士 Wales & 1 \\
美国 United States & 91 & 法国 France & 1 \\
墨西哥 Mexico & 24 & 南极洲 Antarctica & 3 \\
厄瓜多尔 Ecuador & 1 & 日本 Japan & 2 \\
百慕大群岛 Bermuda & 3 & 中国大陆 China mainland & 4 \\
英属维尔京群岛 British Virgin Islands & 3 & 中国台湾 Taiwan, China & 2 \\
苏格兰 Scotland & 1 & 澳大利亚 Australia & 2 \\
爱尔兰 Ireland & 新西兰 New Zealand & 23 \\
\hline
\end{tabular}


性声音的回放设备, 以此来吸引成年燕鸥筑巢繁 殖。在整个繁殖期间岛上监测人员持续不断地对栖 息地进行监控, 并对燕鸥的天敌、竞争者和栖息地 内植被进行管理控制。当年很快就吸引普通燕鸥和 北极燕鸥来此筑巢(Kress, 1998)。到了1981年, 粉红 燕鸥(Sterna dougallii)也被吸引而来筑巢。截至2010 年, 在Eastern Egg Rock岛的人工招引栖息地上繁 殖的燕鸥包括714对普通燕鸥、83对北极燕鸥和82 对粉红燕鸥(Kress et al, 2009)。此后, 相继有大约12 项类似的招引项目在缅因湾实施, 许多海鸟种群得 以回归。

\section{2 美国俄勒 冈州红嘴巨燕鸥 (Hydroprogne caspia)繁殖群的人工迁移}

1997年，鸟类学家发现越来越多的红嘴巨燕鸥 聚集到美国西北部俄勒冈和华盛顿州交界的哥伦 比亚河口的Rice岛进行繁殖(Collis et al, 2002)。在这 里, 这些红嘴巨燕鸥大量捕食同样受胁的大马哈鱼 (Oncorhynchus keta)和虹鳟(Oncorhynchus mykiss)的 幼苗, 引起了渔民和渔业部门的担忧。为了缓解日 益严重的鱼鸟冲突, 1999年俄勒冈州立大学渔业与 野生动物系Daniel Roby教授带领的研究团队在Rice 岛下游 $21 \mathrm{~km}$, 靠近哥伦比亚河口的东沙岛为红嘴 巨燕鸥重建了一块繁殖栖息地。他们把岛边的植被 移除, 露出了下面的沙地(红嘴巨燕鸥喜爱的栖息 环境), 在沙地上布设了太阳能电板和声音回放设 备, 并布置了 380 只假鸟, 以吸引Rice岛上的红嘴巨 燕鸥来东沙岛繁殖。经过两年的时间, 红嘴巨燕鸥 全部从 Rice岛迁移到了东沙岛繁殖(Roby et al, 2002)。在东沙岛繁殖的红嘴巨燕鸥由于更靠近河口 海域, 食物的种类更加多样。1999和2000年, 在Rice 岛繁殖的红嘴巨燕鸥食物中, 大马哈鱼和虹鳟幼苗 的比例分别高达 $77 \%$ 和 $90 \%$, 而1999、2000和 2001 年东沙岛繁殖的红嘴巨燕鸥食物中大马哈鱼和虹 䲡幼苗的比例分别下降到了 $46 \%$ 、47\%和33\%, 而且 红嘴巨燕鸥的繁殖成功率也有了明显的提高(Roby et al, 2002)。东沙岛的燕鸥招引项目, 成功实现了红 嘴巨燕鸥繁殖群从Rice岛到东沙岛的迁移, 大大缓 解了鱼鸟矛盾。

随着红嘴巨燕鸥种群的扩大，它们对鲑鱼资源 造成了威胁。2011年, 又一场红嘴巨燕鸥的大迁移项 目开始实施(Intermountain Communications, 2012)。 这次的项目地点是位于俄勒冈州东南部荒漠地区
的Malheur湖。这是一个内陆的淡水湖, 历史上也曾 经有过红嘴巨燕鸥繁殖的记录。近年来, 亚洲鲤鱼 (包括从亚洲引进的鲤鱼(Cyprinus carpio)、青鱼 (Mylopharyngodon piceus)、草鱼(Ctenopharyngodon idella)、鲫鱼(Carassius auratus)等多种鱼类)在美国 多地泛滥成灾, 其中也包括Malheur湖野生动物保 护区。如何控制亚洲鲤鱼的生长和扩张成了当地管 理部门头痛的问题。东沙岛红嘴巨燕鸥招引的成功 经验给了他们启发, 他们尝试用红嘴巨燕鸥来控制 亚洲鲤鱼的种群扩张。该项目仍由俄勒冈州立大学 的Daniel Roby教授的团队负责实施。他们在湖中央 建造了一个人工岛, 岛上铺设了沙子适于红嘴巨燕 鸥的繁殖栖息，并配置了假鸟、声音回放设备和人 工监测木屋。项目实施的第一年即成功地从哥伦比 亚河口吸引了大群的红嘴巨燕鸥前来栖息繁殖。 Malheur湖的招引成功不仅意味着红嘴巨燕鸥种群 和栖息地的扩大，也意味着哥伦比亚河口鲑鱼的增 加以及Malheur湖亚洲鲤鱼的减少。最近几年，东沙 岛和Malheur湖成功的经验在俄勒冈州和加利福尼 亚州得到了更多的推广, 是野生动物管理特别是解 决鱼鸟之争或利用鸟类控制鱼类扩张的成功典范 (Bird Research Northwest, 2015)。

\section{3 新西兰Mana岛穴居海鸟和岛屿生态系统的恢复}

新西兰的许多海岛曾经是穴居海鸟(如獲科、海 燕科和鹈燕科鸟类)的繁殖栖息地。然而, 由于人为 影响, 许多海鸟逐渐从这些海岛上消失。这不仅导 致了世界上近半数的獲类处于濒危状态, 更有 11 种 甚至达到了极度濒危的程度(Croxall et al, 1984)。繁 殖海鸟的区域灭绝所影响的不仅是海鸟本身, 同时 也对所处的岛屿生态系统造成了严重的影响。穴居 海鸟通过筑巢穴居和踩踏植物改变岛屿的地表和 植被，并通过粪便、呕吐物、弃蛋和尸体等将海洋 营养物转移到陆地上(Smith, 1976), 很大程度上影 响了岛屿生态系统, 包括植被、无脊椎动物、爬行 类和鸟类(Hawke et al, 1999)。Mana岛曾经有多种穴 居海鸟繁殖, 但由于外来种入侵和农业生产活动, 海鸟从Mana岛上消失了。为了恢复海鸟及岛屿生态 系统, 海岛管理部门决定在Mana岛实施穴居海鸟 的恢复项目(Miskelly, 1999)。项目从1993年开始，首 先招引的是鹈燕(Pelecanoides urinatrix)。他们先在 岛上播放鹈燕的叫声, 1997-1999年间, 连续从其他 繁殖地转移了239只雉鸟到Mana岛上(Miskelly \& 
Taylor, 2004)。到2004年, 有20只曾经在Mana岛饲 育长大离开的鹈燕回到了Mana岛。2008年, 至少有 10对鹈燕在岛上繁殖。从1997-2008年, 他们一共在 岛上进行了 8 种鸟蒦类的人工招引, 其他还包括仙锯 鸟蒦 (Pachyptila turtur)、灰脸圆尾獲 (Pterodroma macroptera)、新西兰圆尾獲(P. pycrofti)、查岛圆尾 獲( $P$. axillaris)、红圆尾獲( $P$. magentae $) 、$ 棕嘴鸟蒦 (Puffinus gavia)和澳洲鸟蒦(P. huttoni)。共有 1,791 只雏 鸟转移到Mana岛的人工巢穴进行喂养, 有 1,546 只 长大离岛。截至2009年, 至少已有68只鸟类性成熟 后返回Mana岛繁殖(Miskelly et al, 2009)。

\section{4 浙江韭山列岛极危鸟类中华凤头燕鸥的人工 招引和种群恢复}

中华凤头燕鸥是燕鸥科鸟类中数量最少的种 类, 全球种群数量不到 50 只(Delany \& Scott, 2002), 被IUCN列为极危物种。中华凤头燕鸥在1937年青 岛采集到最后标本之后长达60多年销声匿迹, 直到 2000年在马祖列岛意外被发现混群在大群大凤头 燕鸥 (Thalasseus bergii)中进行繁殖 (Liang et al, 2000)。2004年浙江自然博物馆陈水华等(2005)在浙 江韭山列岛考察时发现了中华凤头燕鸥的繁殖群 体, 该繁殖群体在 2007 年因遭遇人为捡蛋后离开 (Chen et al, 2009, 2010)。为了有效保护中华凤头燕 鸥繁殖群体, 2013年, 浙江自然博物馆联合美国俄 勒冈州立大学和象山县海洋与渔业局在非山列岛 实施了中华凤头燕鸥和大凤头燕鸥种群的人工招 引和恢复项目。项目组选定铁墩岛作为招引栖息地, 经清理和改造, 模拟了中华凤头燕鸥和大凤头燕鸥 的繁殖生境, 布设假鸟模型和鸟声回放音响设备, 并在铁墩岛对面的积谷山设营地观察。当年成功吸 引了19只中华凤头燕鸥和2,000多只大凤头燕鸥来 此繁殖, 最终有 1 只中华凤头燕鸥倠鸟和 600 多只大 凤头燕鸥繁殖成功离岛。2014年开始, 在铁墩岛修 建观察和生活营地, 监测人员进驻招引岛屿, 实现 了全天候监测。2014年有13对中华凤头燕鸥和1,000 多对大凤头燕鸥繁殖成功, 2015年被招引并成功繁 殖的中华凤头燕鸥上升到 16 对, 大凤头燕鸥 1,000 对(陈水华等, 2015)。连续3年的招引成功对中华凤 头燕鸥种群数量的恢复具有重大意义。

\section{5 讨论}

海鸟种群受胁和栖息地的丧失是一个世界性
的问题, 关于如何恢复海鸟种群及其栖息地, 欧美 国家尤其是北美较早开展了这方面的探索。雏鸟转 移和社群吸引等人工招引技术就是这一探索的重 要成果。海鸟种群的人工招引和栖息地恢复技术的 应用, 为受威胁和濒临灭绝的海鸟带来了福音。尤 其是社群吸引技术可以在短时间内有效恢复海鸟 种群数量并扩大其生存范围, 这对于那些种群数量 减少、生存范围缩小、繁殖栖息地被破坏的燕鸥科 种群的恢复具有良好的应用前景。

\section{1 社群吸引技术的应用要点和局限}

成功的社群吸引技术需要多方面的保证: 一是 对消失不久的物种进行招引, 这样可以充分了解其 栖息环境; 二是必须有合适的岛屿用于招引，不同 种类的海鸟繁殖所需的栖息环境是不同的, 包括各 种地表条件、洞穴、植被覆盖度和高度、树木等因 素, 所选岛屿必须具备可供改造的条件; 三是要有 足够的时间来提前布置整个栖息地, 由于假鸟模型 诱饵通常只对昼行性海鸟有作用, 而声音对昼行性 和夜行性海鸟都有作用, 所以社群吸引技术通常需 要用假鸟模型与声音相结合的方式来吸引海鸟, 这 就需要在海鸟繁殖期到来之前有充足时间摆放假 鸟模型以及声音回放设备; 四是要在布置完成栖息 地以后清除整个岛上所有对海鸟有威胁的物种, 并 在繁殖期内监测是否有遗漏的危险物种和猛禽; 五 是针对不同海鸟和不同繁殖时期制作并布设不同 的假鸟模型并调整模型密度, 如需要也可在栖息地 内布设镜子, 通过反射增加假鸟的密度; 六是在整 个繁殖期, 岛上监测人员需要不间断观察, 随时监 控各种条件的变化, 并根据情况及时解决问题 (Parker et al, 2007)。

比较而言, 单纯使用假鸟和单纯使用声音回放 招引的成功率并不高, 分别仅为 $44 \%$ 和 $42 \%$ 。如果将 两者结合, 则成功率可达 $80 \%$ 以上。

虽然社群招引技术成功的案例很多, 但是其局 限性也比较明显。这项技术目前多用于对燕鸥的招 引恢复项目, 并且多数取得了成功。这是因为燕鸥 对假鸟模型和声音的反应比其他海鸟更迅速, 往往 第一年就可以招引成功; 再者, 燕鸥很少显示出对 出生地的依恋, 这就方便在新的栖息地上进行招引 (Jones \& Kress, 2012)。燕鸥种类多、分布广, 一个 项目的成功会带动类似项目的实施。社群吸引技术 应用在其他海鸟的招引上不那么成功, 主要是因为 
其他海鸟对模型或者声音的反应不够迅速, 往往需 要较长时间来适应新的栖息地, 使得整个项目周期 较长, 容易出现资金困难。所以, 应用社群吸引技 术招引海鸟是一个缓慢的过程, 政府或有关组织的 重视和资金的供给是整个项目中最重要的方面。

\section{2 雏鸟转移技术的应用要点和局限}

比较而言, 雏鸟转移一般需要 5 年以上才能初 见成效, 十多年甚至更长时间的监测才能确认成效, 对于那些性成熟较晚的海鸟尤其如此。缅因湾的北 极海鹦招引, 4 年之后才有当年的海鹦回归, 8 年之 后才有第一个个体繁殖, 35年之后, 繁殖对才达到 100 。因而雉鸟转移技术需要有长久的计划和足够 的耐心。其次, 雏鸟转移对栖息地有特殊的要求, 由于适于倠鸟转移的多属于穴居海岛, 目标岛屿上 应有适于筑巢繁殖的洞穴。此外, 雏鸟转移要求人 工饲育雏鸟, 针对不同的海鸟采取针对性的人工饲 育技术, 以免造成维鸟不必要的死亡。

\section{3 我国海鸟人工招引和种群恢复展望}

在实施海鸟人工招引项目中, 应针对拟招引的 鸟类及其栖息地开展较为具体的前期研究, 包括目 标鸟类的栖息地类型、巢位选择对策、繁殖时间表 和基本繁殖信息和行为等。所选择的栖息地一定要 适合于目标鸟类的繁殖和栖息, 周边环境和食物资 源应可以满足整个繁殖群的需要。必须综合考虑栖 息地适应性、食物资源、人为干扰、天敌风险以及 工作便利等多种因素。特别是应根据目标鸟类的生 态习性选择适合的招引方法, 切忌盲目开展。此外, 在不同地区开展招引, 天敌风险不尽相同。虽然猛 禽、蛇和鼠类等动物是海鸟最普遍的威胁因素, 但 针对具体项目, 仍应开展具体的调研。条件允许的 话, 近距离全天候的监测, 可以有效掌握天敌等威 胁因素。在项目实施过程中, 及时了解鸟类的动态, 排除威胁因子, 是海鸟招引成功的关键。

中国拥有漫长的海岸线, 跨越亚热带和温带气 候区, 众多的无人岛屿可以为海鸟提供良好的繁殖 和栖息的场所。根据现有的文献和调查资料, 在我 国海域岛屿繁殖的海鸟计有 4 目13科25种(陈水华, 2010)。其中包括白额鸟蒦(Calonectris leucomelas)、黑 叉尾海燕(Oceanodroma monorhis)、海胪鹚(Phalacrocorax pelagicus)、黑尾鸥(Larus crassirostris)、中 华凤头燕鸥、大凤头燕鸥、粉红燕鸥、黑枕燕鸥 (Sterna sumatrana)、褐翅燕鸥(S. anaethetus)、白顶
玄燕鸥(Anous stolidus)和扁嘴海雀(Synthliboramphus antiquus)等。然而, 由于我国沿海普遍存在人为 干扰、海洋污染、过度捕捞、台风、鼠害和蛇害等 多种威胁因素, 海鸟的繁殖区域和岛屿在缩减, 包 括中华凤头燕鸥等多种国际受胁物种和国家重点 保护物种在内的海鸟种群数量明显下降(陈水华, 2010)。目前, 应用社群吸引技术在浙江韭山列岛实 施的大凤头燕鸥和中华凤头燕鸥人工招引和种群 恢复技术取得了初步成功。社群吸引和倠鸟转移等 海鸟人工招引技术在北美、欧洲和新西兰等地被广 泛应用, 并取得显著成效。这些技术的应用对于我 国沿海海鸟的种群及其栖息地的恢复具有重要的 借鉴意义。

致谢：感谢墨西哥岛屿生态保护组织的Yuliana Rocío Bedolla Guzmán博士和美国俄勒冈州立大学 的Daniel Roby教授提供部分资料和信息。

\section{参考文献}

Bird Research Northwest (2015) Monitoring and Predator Control at the Corps-Constructed Caspian Tern Islands in Southeastern Oregon and Northeastern California, 2015 Annual Report. http://www.birdresearchnw.org/2015\%20Predator\%20Control\%20Final\%20Report_v12.pdf. (accessed on 2016-12-22)

Chen SH (2010) Status and protection of the breeding seabirds in China Seas. Bulletin of Biology, 45(3), 1-4. (in Chinese) [陈水华 (2010) 中国海域繁殖海鸟的现状与保护. 生物 学通报, 45(3), 1-4.]

Chen SH, Fan ZY, Lu YW (2015) The population of the Chinese crested tern is close to one hundred. http://www.chinabird.org/news/chinese\%20tern\%202015.htm. [陈水华, 范 忠勇, 陆祎玮 (2015) 中华凤头燕鸥招引繁殖再获成功, 种群数量接近百只. http://www.chinabird.org/news/chinese \%20tern\%202015.htm.] (accessed on 2016-12-22)

Chen SH, Chang SH, Liu Y, Chan S, Fan ZY, Chen CS, Yen CW, Guo DS (2009) Low population and severe threats: status of the critically endangered Chinese crested tern Sterna bernsteini. Oryx, 43, 209-212.

Chen SH, Fan ZY (2013) Chinese crested tern. Chinese Journal of Zoology, 48, 788-789. (in Chinese) [陈水华, 范忠勇 (2013) 中华凤头燕鸥. 动物学杂志, 48, 788-789.]

Chen SH, Fan ZY, Chen CS, Lu YW, Wang ZD (2010) A new breeding site of the critically endangered Chinese crested tern Sterna bernsteini in the Wuzhishan Archipelago, eastern China. Forktail, 26, 132-134.

Chen SH, Yan CW, Fan ZY, Chen CS, Zhang FG (2005) The breeding colony of Chinese crested tern at Jiushan Archipelago 
in Zhejiang. Chinese Journal of Zoology, 10(1), 96-97. (in Chinese) [陈水华, 颜重威, 范忠勇, 陈苍松, 张方刚 (2005) 浙江韭山列岛的黑嘴端凤头燕鸥繁殖群调查初 报. 动物学杂志, 10(1), 96-97.]

Collis K, Roby DD, Craig DP, Adamany S, Adkins JY, Lyons DE (2002) Colony size and diet composition of piscivorous waterbirds on the lower Columbia River: implications for losses of juvenile salmonids to avian predation. Transactions of the American Fisheries Society, 131, 537-550.

Croxall JP, Prince PA, Hunter I, McInnes SJ, Copestake PG (1984) The seabirds of the Antarctic Peninsula, Islands of the Scotia Sea and Antarctic Continent between $80^{\circ} \mathrm{W}$ and $20^{\circ} \mathrm{W}$ : their status and conservation. ICBP Technical Publication, 2, 637-666.

Croxall JP, Trathan PN, Murphy EJ (2002) Environmental change and Antarctic seabird populations. Science, 297, 1510-1514.

Delany S, Scott D (2002) Waterbird Population Estimates, 3rd edn. Wetland International, Wageningen, The Netherlands.

Donlan CJ, Wilcox C (2007) Complexities of costing eradications. Animal Conservation, 10, 154-156.

Frederiksen M, Harris MP, Daunt F, Rothery P, Wanless S (2004) Scale-dependent climate signals drive breeding phenology of three seabird species. Global Change Biology, 10, 1214-1221.

Gaston AJ (2004) Seabirds: A Natural History. Yale University Press, New Haven.

Hasegawa H, Watkinson AR (1982) The short tailed albatross, Diomedea albatrus, its status, distribution and natural history. American Birds, 36, 806-814.

Hawke DJ, Holdaway RN, Causer JE, Ogden S (1999) Soil indicators of pre-European seabird breeding in New Zealand at sites identified by predator deposits. Australian Journal of Soil Research, 37, 103-113.

Howald G, Donlan CJ, Tershy BR, Croll DA, Russell J, Saunders A, Clout $M$ (2007) Invasive rodent eradications on islands. Conservation Biology, 21, 1258-1268.

Intermountain Communications (2012) New man-made island in Malheur Lake attracting Caspian terns from estuary's East Sand Island. The Columbia Basin Fish \& Wildlife News Bulletin. http://www.cbbulletin.com/421296.aspx. (accessed on 2016-12-22)

IUCN (International Union for Conservation of Nature) (2009) The IUCN Red List of Threatened Species. http://www.iucn redlist.org. (accessed on 2016-12-22)

Jones HP, Kress SW (2012) A review of the world's active seabird restoration projects. The Journal of Wildlife Management, 76, 2-9.

Jones HP, Towns DR, Bodey T, Miskelly CM, Ellis J, Rauzon MJ, Kress SW, McKown M (2011) Recovery and restoration on seabird islands. In: Seabird Islands: Ecology, Invasion, and Restoration (eds Mulder CPH, Anderson WB, Towns DR, Bellingham PJ), pp. 460-531. Oxford University Press, Oxford.

Kress SW (1983) The use of decoys, sound recordings and gull control for re-establishing a tern colony in Maine. Colonial Waterbirds, 6, 185-196.

Kress SW (1997) Using animal behavior for conservation: case studies in seabird restoration from the marine coast, USA. Journal of the Yamashina Institute for Ornithology, 29, 1-26.

Kress SW (1998) Applying research for effective management: case studies in seabird restoration. In: Avian Conservation: Research and Management (eds Marzluff JM, Sallabanks R), pp. 141-154. Island Press, Washington, DC.

Kress SW, Borzik RV, Hall CS (2009) Egg Rock Update 2009. National Audubon Society, Ithaca, New York.

Kress SW, Nettleship D (1988) Re-establishment of Atlantic puffins (Fratercula arctica) at a former breeding site in the gulf of Maine. Journal of Field Ornithology, 59, 161-170.

Liang CT, Chang SH, Fang WH (2000) Little known oriental bird: discovery of a breeding colony of Chinese crested tern. Oriental Bird Club Bulletin, 32, 18.

Miskelly CM (1999) Social constraints on access to mates in a high density population of New Zealand Snipe (Coenocorypha aucklandica). Notornis, 46, 223-239.

Miskelly CM, Gummer H (2013) Attempts to anchor pelagic fairy prions (Pachyptila turtur) to their release site on Mana Island. Notornis, 60, 29-40.

Miskelly CM, Taylor GA (2004) Establishment of a colony of Common diving petrels (Pelecanoides urinatrix) by chick transfers and acoustic attraction. Emu, 104, 205-211.

Miskelly CM, Taylor GA, Gummer H, Williams R (2009) Translocations of eight species of burrow-nesting seabirds (genera Pterodroma, Pelecanoides, Pachyptila and Puffinus: Family Procellariidae). Biological Conservation, 142, 1965-1980.

Nogales M, Martin A, Tershy B, Donlan CJ, Veitch D, Puerta N, Wood B, Alonso J (2004) A review of feral cat eradication on islands. Conservation Biology, 18, 310-319.

Parker MW, Kress SW, Golightly RT, Carter HR, Parsons EB, Schubel SE, Boyce JA, McChesney GJ, Wisely SM (2007) Assessment of social attraction techniques used to restore a common murre colony in central California. Waterbirds, 30, 17-28.

Roby DD, Collis K, Lyons DE, Craig DP, Adkins JY, Myers AM, Suryan RM (2002) Effects of colony relocation on diet and productivity of Caspian terns. Journal of Wildlife Management, 66, 662-673.

Rolland C, Danchin E, Fraipont MD (1998) The evolution of coloniality in birds in relation to food, habitat, predation, and life-history traits: a comparative analysis. The American Naturalist, 151, 514-529.

Schreiber EA, Burger J (2001) Biology of Marine Birds. CRC Press, Boca Raton.

Smith VR (1976) The effect of burrowing species of Procellariidae on the nutrient status of inland tussock grasslands on Marion Island. Journal of South African Botany, 42, 265-272.

(责任编委: 丁平 责任编辑: 时意专) 